\title{
Digital Fresnel Hologram Watermarking
}

\author{
Naveen K. Nishchal \\ Department of Physics \\ Indian Institute of Technology \\ Patna, Patna-800 013, India \\ E-mail: nknishchal@iitg.ernet.in
}

\author{
Tomi Pitkäaho \\ RFMedia Laboratory \\ Oulu Southern Institute \\ University of Oulu, 84100 \\ Ylivieska, Finland \\ E-mail: tomi.pitkaaho@oulu.fi
}

\author{
Thomas J. Naughton \\ Department of Computer \\ Science, National University of \\ Ireland Maynooth, Ireland \& RF \\ Media Laboratory, Oulu \\ Southern Institute, University of \\ Oulu, Ylivieska, Finland \\ E-mail: tomn@,cs.nuim.ie
}

\begin{abstract}
We present a method of digital hologram watermarking using a Fresnel hologram of a real-world 3D object and the fractional Fourier transform. A watermark is encrypted using double random phase fractional Fourier domain encoding technique and then encoded into the digital hologram. The hologram is watermarked in a plane at some known distance from the object so that even if a new hologram is generated from the original hologram the watermark can always be traced by propagating the new hologram back to the object and then onto the watermark plane. The watermark is retrieved successfully using the correct encryption parameters. We consider both numerical (full complex field) and optoelectronic (phase-only) reconstruction methods. We obtain the watermark from different windows of the hologram corresponding to different reconstruction perspectives.
\end{abstract}

\section{INTRODUCTION}

Digital holography (DH) refers to the science of using discrete electronic devices, such as CCDs to record the hologram. In this case, the reconstruction can be performed numerically by simulating the propagation of the wavefield back to the plane of the object. With advances in computer performance and electronic image acquisition devices, $\mathrm{DH}$ is becoming more attractive for many applications [1-6]. Storage of the hologram in a computer enables us to reduce the noise through image processing techniques and numerically reconstruct the object with arbitrary views. The technique also makes it possible to record video holograms of a $3 \mathrm{D}$ object. It is possible to generate another hologram with one optically recorded or computer generated holograms by propagating the hologram in free-space at certain distances. Thus, the original holographer may lose his ownership if somebody generates a new hologram and claims his/her right. This allows unwanted use of the holographic information, which we may call Hologram Piracy. Piracy of information without appropriate permission from rightful owners deprives the original creators of their rights. It is therefore desirable in some quarters to protect the individual holographer's ownership/rights. A possible solution to protect from hologram piracy could be embedding a watermark into the hologram. Watermarking of 2D or $3 \mathrm{D}$ data has been extensively studied to provide protection for digital image, audio, and video [1-9].

A watermark is a visible or invisible identification code that is permanently embedded in the data and remains present within the data after any decryption process [1]. A transformation domain is often needed for robustly embedding an invisible watermark, for example the frequency domain. Therefore, the signal energy present in any signal frequency becomes undetectable.

Watermarking in a fractional order domain provides extra security against attackers compared to the spatial or spatial frequency domains since the fractional orders of the transform provides extra degree of freedom [6,8]. Properties and applications of the ordinary Fourier transform are special cases of those of the fractional Fourier transform (FRT), which is a generalization of the ordinary Fourier transform with an order parameter $\alpha$ [6]. The generalization of the ordinary Fourier transform to the FRT comes at no additional cost in digital computation or optical implementation. In this paper, we present a method of watermarking a real phaseshift Fresnel digital hologram of a real world threedimensional (3D) scene using the FRT. The watermark is encrypted using the double random phase encoding technique. The encrypted watermark is embedded into the digital hologram at a specified location relative to the object and can always be recovered by first propagating the newly generated hologram back to the object location. We consider both numerical (full complex field) and optoelectronic (phase-only) reconstruction methods. We also obtain the watermark from different reconstruction perspectives.

\section{PRINCIPLE}

We followed the conventional double random phase encoding scheme for encryption of the watermark. The random phase masks were placed in the fractional Fourier plane instead of the 
conventional Fourier plane. Let function $f(x, y)$ represent the watermark to be encrypted by double random fractional Fourier domain encoding scheme. The watermark is multiplied with a random phase mask (RPM1), defined as $\exp [2 \pi j r(\xi, \eta)]$, and its FRT of order $\alpha$ is obtained. A two-dimensional FRT of function $\{f(x, y) \times \exp [2 \pi j r(\xi, \eta)]\}$ of order $\left(\alpha_{1}=\mathrm{p}_{1} \pi / 2\right)$ is given by $g(\xi, \eta)$ as [6]

$$
\begin{aligned}
g(\xi, \eta) & =K \iint f(x, y) \times \exp [2 \pi j r(x, y)] \\
& \times \exp \left(j \pi \frac{x^{2}+y^{2}+\xi^{2}+\eta^{2}}{\tan \alpha_{1}}-2 j \pi \frac{x y \xi \eta}{\sin \alpha_{1}}\right) d x d y
\end{aligned}
$$

Here $(x, y)$ and $(\xi, \eta)$ represent the space and fractional domain coordinates, respectively. The parameter $\mathrm{K}$ is a complex constant. The function $g(\xi, \eta)$ is multiplied by another mask, RPM2, defined as $\exp [2 \pi j r(\rho, \sigma)]$, and an FRT of order $\left(\alpha_{2}=\mathrm{p}_{2} \pi / 2\right)$ is obtained, which gives the encrypted image as

$$
\begin{aligned}
e(\rho, \sigma)= & \left.K \iint\{g(\xi, \eta) \times \exp 2 \pi j r(\xi, \eta)]\right\} \\
& \times \exp \left(j \pi \frac{\xi^{2}+\eta^{2}+\rho^{2}+\sigma^{2}}{\tan \alpha_{2}}-2 j \pi \frac{\xi \eta \rho \sigma}{\sin \alpha_{2}}\right) d \xi d \eta
\end{aligned}
$$

The encrypted image of watermark, $e(\rho, \sigma)$, is combined with the Fresnel digital hologram, $h(\rho, \sigma)$. The watermarked image, $w(\rho, \sigma)$, is then given by

$$
w(\rho, \sigma)=h(\rho, \sigma)+a e(\rho, \sigma)
$$

where $a$ is an arbitrary constant, called the weighting factor of the watermark. It has been shown that the optimal weighting factor produces the least errors in the reconstructed 3D host object and the decoded watermark even in the presence of an occlusion attack [4].

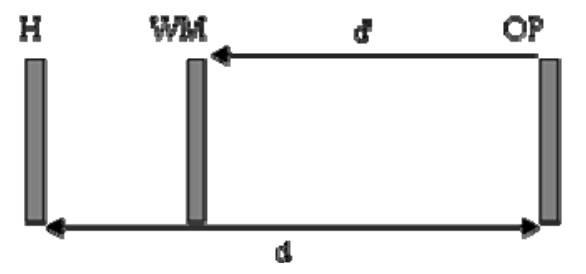

Fig. 1. Schematic to show the different planes. H, hologram plane; WM, watermarking plane; OP, an object plane; d, separation between the hologram plane and object; $d$, separation between the object and the watermarking plane.

Figure 1 shows the schematic of the different planes. For better security purposes we encoded the watermark in a plane that was a known distance $d^{\prime}$ from the object. One may encode the watermark in the hologram plane but in that case if a new hologram is generated then it is not possible to recover the watermark without an exhaustive search. In this proposal, the search space is reduced to the uncertainty of determining the front of the 3D object in the reconstruction volume.

\section{EXPERIMENT}

The results of computer experiment carried out using the MATLAB platform are shown in Figs. 2(ah) and 3(a-d). We used a phase-shift digital hologram of a real world 3D scene recorded with parameters $\lambda$ $=632.8 \mathrm{~nm}$, recording distance, $\mathrm{d}=179 \mathrm{~mm}$, and pixel size $=7.4 \mu \mathrm{m} \times 7.4 \mu \mathrm{m}$. The object, a chip, was used for the study. The recorded digital hologram is of size $2048 \times 2048$ pixels, as shown in Fig. 2(a). The reconstruction is shown in Fig. 2(b). The watermark, as shown in Fig. 2(c) is encrypted with two random phase masks placed at input and the fractional domains, respectively. The fractional orders used for encryption were $\mathrm{p}_{1}=0.555$ and $\mathrm{p}_{2}=0.355$. The encrypted image of the watermark was embedded (a $=0.25)$ to the regenerated hologram at $\mathrm{d}^{\prime}=20 \mathrm{~mm}$ distance from the original hologram plane and has been shown in Fig. 2(d). The recovered watermark after using the correct encryption keys is shown in Fig. 2(e). Fig. 2(f) shows the intensity image when we tried to recover the watermark with one wrong fractional order $\mathrm{p}_{1}=0.50$ and using other fractional order and RPMs correctly. The watermarked hologram was also reconstructed and the reconstructed intensity image has been shown in $2(\mathrm{~g})$.

It is not yet possible to reliably and conveniently modulate a spatial-light modulator with arbitrary complex-values. Phase-modulating devices are often used to optoelectronically reconstruct appropriately modified complex-valued holograms. We consider only the phase of the watermarked complex-valued hologram by setting each amplitude to unity as shown in Fig. 2(g) and successfully recover the watermark from the degraded hologram as shown in Fig. 2(h). We also obtained the watermark from different windows of the hologram corresponding to different reconstruction perspectives. The results have been shown in Figs. 3(a-d). We cropped only $25 \%$ of the watermarked hologram from the right side, as shown in Fig. 3(a) and recovered the watermark, as shown in Fig. 3(b). The cropping from left side and correspondingly recovered watermark have been shown in Figs. 3(c) and (d), respectively.

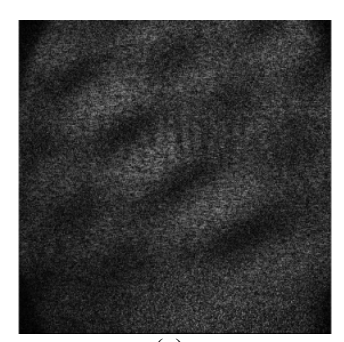

(a)

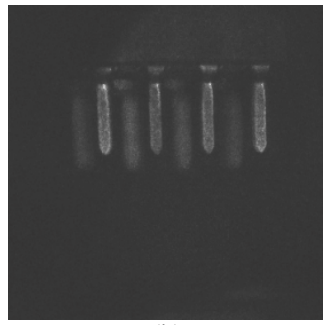

(b) 


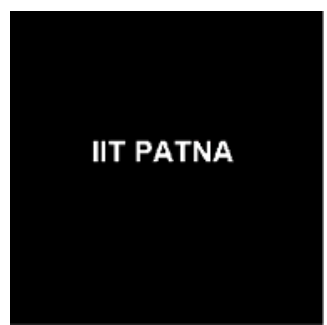

(c)

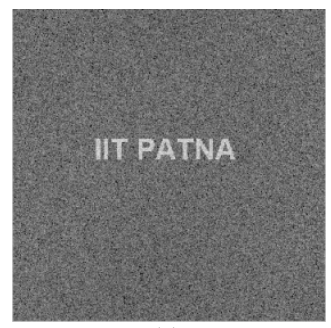

(e)

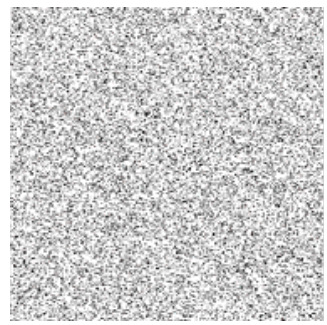

(g)

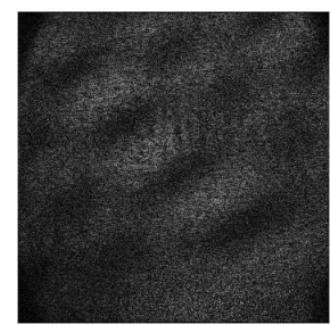

(d)

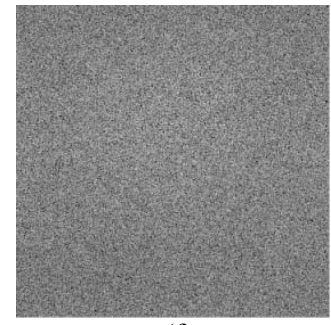

(f)

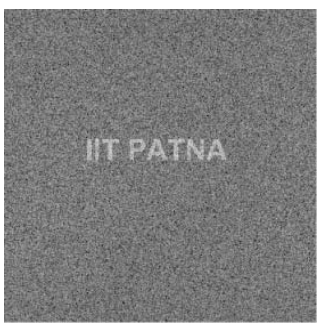

(h)
Fig. 2 (a) phase-shift Fresnel digital hologram, (b) reconstructed object, (c) watermark, (d) watermarked digital hologram, (e) recovered watermark, (f) recovered watermark with one of the wrong fractional orders, (g) phase of the hologram only, and (h) recovered watermark with phase-only hologram.

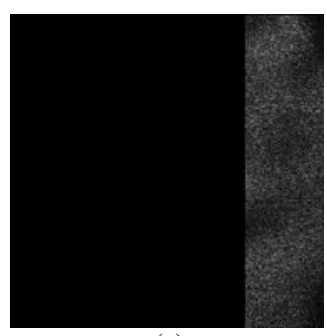

(a)

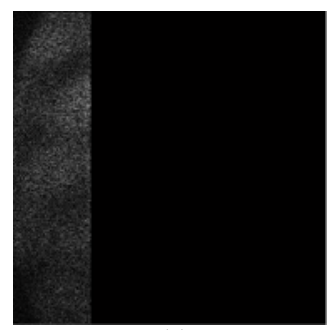

(c)

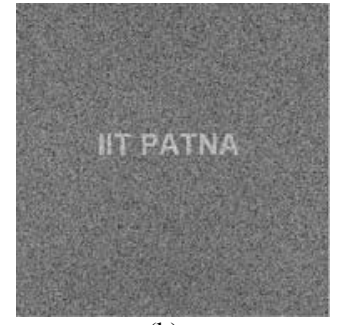

(b)

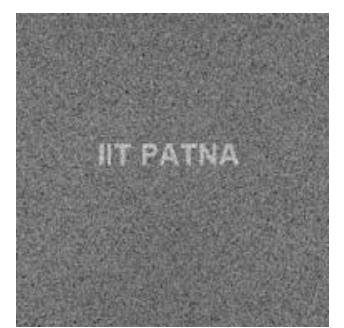

(d)
Fig. 3 (a) cropped $25 \%$ from right side of the watermarked hologram, (b) recovered watermark, (c) cropped $25 \%$ from left side of the watermarked hologram, and (d) recovered watermark.

\section{CONCLUSION}

A method to protect from hologram piracy has been presented that employs watermarking a digital hologram at a specific plane from the sensed object. We demonstrate the technique with a phase-shift Fresnel digital hologram of a real world 3D scene and the FRT. The watermark can always be recovered by propagating the watermarked hologram back to the object and then to a well-known plane before applying the correct decryption keys. We also recover the watermark from a phase-encoded version of the digital hologram. We obtain the watermark from different windows of the hologram corresponding to different reconstruction perspectives of the $3 \mathrm{D}$ scene.

\section{ACKNOWLEDGEMENT}

The authors thank Emmanouil Darakis for capturing the hologram. Support is acknowledged from the Academy of Finland, Science Foundation Ireland under the National Development Plan, and the European Commission through a Marie Curie Fellowship.

\section{REFERENCES}

[1] B. Javidi, Ed., Optical Imaging Sensors and Systems for Homeland Security Applications, Springer: New York, 2006.

[2] S. Kishk and B. Javidi, "Watermarking of three-diemnsional objects by digital holography," Opt. Lett., vol. 28, pp. 167$169,2002$.

[3] S. Kishk and B. Javidi, "3D watermarking by a 3D hidden object," Opt. Express, vol.11, pp. 874-888, 2003.

[4] H. Kim and Y. H. Lee, "Optimal watermarking of digital hologram of 3-D object," Opt. Express, vol. 13, pp. 28812886, 2005.

[5] X.-F. Meng, L.-Z. Cai, X.-L. Yang, X.-F. Xu, G.-Y. Dong, X.-X. Shen, H. Zhang, and Y.-R. Wang, "Digital color image watermarking based on phase-shifting interferometry and neighboring pixel value subtraction algorithm in the discretecosine-transform domain," Appl. Opt., vol. 46, pp. 46944701, 2007.

[6] N. K. Nishchal and T. J. Naughton, "Three-dimensional image watermarking using fractional Fourier transform," Proc. of Int'l Confer. on Optics and Photonics, CSIO Chandigarh, Oct. 30-Nov. 1, 2009.

[7] L. Sun and S. Zhuang, "Watermarking by encrypted Fourier holography,” Opt. Eng., vol. 46, pp. O85801, 2007.

[8] I. Djurovic, S. Stankovic, and I. Pitas, "Digital watermarking in the fractional Fourier transformation domain," J. Network Computer Appl., vol. 24, pp. 167-173, 2001.

[9] K. Deng, G. Yang, and C. Zhang, "Burch computergenerated hologram watermarking resilient to strong cropping attack," Biomed. Opt., OSA Tech. Digest, JMA 30, 2010. 